\title{
Diacronie
}

Studi di Storia Contemporanea

$N^{\circ} 12,4 \mid 2012$

Sulle tracce delle idee

\section{Le radici ottomane della Turchia di Erdoğan}

\section{Federico Donelli}

\section{(2) OpenEdition}

\section{Journals}

\section{Edizione digitale}

URL: http://journals.openedition.org/diacronie/2545

DOI: $10.4000 /$ diacronie. 2545

ISSN: 2038-0925

\section{Editore}

Association culturelle Diacronie

\section{Notizia bibliografica digitale}

Federico Donelli, « Le radici ottomane della Turchia di Erdoğan », Diacronie [Online], N 12, 4 | 2012 ,

documento 9, Messo online il 29 décembre 2012, consultato il 11 juin 2020. URL : http://

journals.openedition.org/diacronie/2545; DOI : https://doi.org/10.4000/diacronie.2545 


\section{Diacronie}

9/

\section{Le radici ottomane della Turchia di Erdoğan}

Federico DONELLI*

L'articolo analizza due fasi della recente storia turca, l'ascesa di Turgut Özal (1982) e l'attuale governo a guida Erdoğan, dalle cui rispettive politiche emergono elementi volti ad un comune richiamo identitario al passato ottomano. Definita da molti analisti come una politica di stampo neo-ottomano, questa ha iniziato a svilupparsi negli anni Ottanta parallelamente ad un altro fenomeno, ossia, il riemergere dell'Islam nel contesto pubblico e politico. Sviluppi favoriti dalle scelte intraprese da Özal che per primo cercò di rilanciare le ambizioni turche attraverso una graduale riconciliazione con l'eredità imperiale. Idee riprese e sviluppate nell'ultimo decennio dall'azione politica del Primo Ministro Erdoğan e del suo ideologo, il Ministro degli Esteri Davutoğlu, i quali hanno rilanciato nel contesto internazionale l'idea neo-ottomana della Turchia convinti che possa far rivivere i fasti di allora, facendole riassumere il ruolo di potenza regionale e globale.

\section{Introduzione}

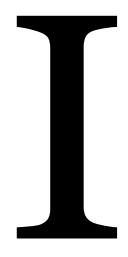

1 Medio Oriente si presenta oggi come una regione sempre più confusa ed instabile, attraversata da eventi rivoluzionari (la cui direzione è ancora indecifrabile) in cui si scontrano, come già accaduto in passato, istanze modernizzatrici e forti richiami alla tradizione islamica.

La crisi finanziaria che negli ultimi anni ha colpito le principali economie occidentali, portandole ad una contrazione della propria presenza nella regione, ha favorito l'emergere di nuovi attori nel contesto geopolitico mediorientale: tra questi spicca una Turchia sempre più desiderosa di recuperare il proprio ruolo di potenza. 
Proponendosi al mondo musulmano come modello di democrazia, la Turchia prova a scalfire definitivamente la diffidenza del mondo arabo che la percepisce ancora come l'erede del dispotismo caratteristico dell'Impero ottomano.

Centrale in questo processo è la politica estera turca che, dopo anni di cauto isolamento, ha trovato le proprie linee guida nella dottrina geopolitica di Ahmet Davutoğlu, conosciuta come "profondità strategica".

Una dottrina generata e successivamente applicata in un contesto caratterizzato dal Neo-ottomanesimo ovvero dal richiamo all'ideologia politica ottocentesca conosciuta come ottomanismo.

Il neo-ottomanesimo, tuttavia, non può considerarsi semplice ideologia, ma piuttosto un generale modo di vivere e pensare la Turchia moderna del presente e del futuro richiamandosi ad un passato glorioso ed ambizioso, ritenuto per decenni scomodo e perdente. Il neo-ottomanesimo, nondimeno, è un termine ancora poco accettato ed utilizzato dall'establishment politico turco che ufficialmente non riconosce le proprie politiche come tali nel timore che ciò possa suscitare richiami errati ad un periodo di neo-imperialismo ${ }^{1}$.

Questo termine, infatti, introdotto alla metà degli anni Ottanta per definire le crescenti ambizioni geo-politiche della nuova Turchia - avvalorate dal recupero dell'eredità ottomana - si presenta ancora come un fenomeno eminentemente accademico e giornalistico.

Il seguente lavoro si propone di analizzare e comprendere gli sviluppi di questa idea non solamente politica ma anche sociale e civica, cercando di coglierne gli elementi fondativi e, al contempo, di provare a capire dove affonda le proprie radici, pur mantenendo una visione costantemente proiettata sul presente e sui possibili sviluppi futuri.

Nella prima parte verrà analizzato il periodo fondativo del neo-ottomanesimo: gli anni caratterizzati dalla figura di Turgut Özal (1983-1989) e dal sorgere di un sentimento riformatore che segnò un primo, seppur non radicale, distacco dalla politica e dall'identità kemalista favorendo la graduale politicizzazione dell'Islam promossa dalla sintesi turco-islamica.

La seconda parte del lavoro, invece, si soffermerà sull'ascesa e la definitiva affermazione del Neo-ottomanesimo come idea guida - sia in politica interna, sia in

\footnotetext{
${ }^{1}$ Il termine venne proposto da un noto scrittore e giornalista turco Cengiz Çandar, il quale vedeva la Repubblica come la naturale erede dell'Impero ottomano e definì la politica estera del governo Özal come neo-ottomana.
} 
politica estera - sotto l'egida di un partito islamico moderato come l'AKP guidato dall’influente figura del Primo Ministro Recep Tayyip Erdoğan.

Proprio il governo dell'AKP, in carica dal 2002, rappresenta oggi ed in prospettiva la miglior concretizzazione del neo-ottomanesimo dove l'islamismo incontra la democrazia e il progresso ma anche la storia, il passato ed un'identità che sembrava dimenticata.

\section{La vittoria di Özal e l'emergere della sintesi turco-islamica}

L'avvento nel mondo politico turco di Turgot Özal nel 1983 rappresentò l'inizio della rottura con il passato e con la politica dei primi sessant'anni della Repubblica, che era stata improntata sui valori kemalisti ben espressi nel cosiddetto "lungo discorso" di Atatürk all'Assemblea Generale nel 1927 e successivamente ripresi nelle famose "sei frecce”2 programmatiche del 1931.

Özal, chiamato inizialmente (1979) a risanare l'economia del Paese nella veste di superministro delle finanze, vinse inaspettatamente le elezioni del 1983 alla guida del Anavatan Partisi (ANAP) 3 . Le elezioni ebbero una grande rilevanza perché furono le prime dopo il colpo di Stato militare del 1980 e la successiva promulgazione della Costituzione (1982) che, seppur con diverse modifiche, è tuttora vigente. La Turchia stava uscendo da un decennio doloroso, caratterizzato da violenti scontri interni tra forze estreme di destra e di sinistra a cui si aggiunse, tra il 1977 e il 1980, l'emegere della guerriglia armata ad opera del PKK (Partîya Karkerén Kurdîstan4)5.

Le elezioni si svolsero in un clima di generale clima interno di inquietudine ed estremizzazione delle posizioni, dovuto anche all'onda lunga della rivoluzione khomeinista (1979), che influenzò piccoli gruppi radicali turchi allarmando l'esercito garante della laicità dello Stato ${ }^{6}$.

L'ascesa di Özal risulterà decisiva per l'avvio di quel cambiamento che nel giro di dieci anni spingerà la Turchia verso una sempre più decisa trasformazione, tanto in

2 Le "sei frecce sono sei parole (nazionalismo, populismo, repubblicanesimo, statalismo, laicismo, rivoluzionarismo) che rappresentano le linee programmatiche della filosofia kemalista ovvero una equilibrata sintesi di nazional populismo e corporativismo. Principi approvati dal Partito Repubblicano del popolo nel 1931 e integrati nella costituzione del 1937 dando così completa attuazione al regime del partito-stato. Si veda: BERNARDINI D'ARNESANO, Beatrice, CARDUCCI, Michele, Turchia, Bologna, Il Mulino, 2008, pp. 52-54.

3 "Partito della Madrepatria".

4 "Partito dei Lavoratori del Kurdistan".

5 BOZARSLAN, Hamit, La Turchia contemporanea, Bologna, Il Mulino, 2006, p. 84.

${ }^{6}$ Il clima di tensione crebbe ulteriormente pochi giorni prima dell'intervento militare quando nella cittadina di Konya durante un comizio un gruppo islamico invocò il ripristino della Şeriat (Shari'a). 
politica interna, quanto in politica estera, in cui centrale risultò essere la progressiva riabilitazione dell'Islam sia nella sfera privata dei cittadini che in quella pubblica.

Un processo che, dopo un breve stallo negli anni Novanta, conoscerà le sue tappe più significative nel nuovo millennio sotto la guida di Recep Tayyip Erdoğan e del AKP.

Determinanti per il successo di Özal furono diversi fattori, interni ed esterni alla Turchia, come il profondo e preoccupante isolamento dovuto alla crisi cipriota (1974) a cui fece seguito il congelamento dei rapporti con l'alleato americano e con l'Europa.

L'isolamento diplomatico aggravò ulteriormente la già precaria situazione economica del Paese, che nel corso del decennio precedente aveva dovuto fronteggiare un'esorbitante inflazione, la quale, in continuo aumento, nel 1979 raggiunse lo straordinario tasso del $90 \% 7$.

Politicamente, invece, fu determinante il golpe militare del 1980 che, deposto il governo, sospese tutti i partiti politici e arrestò i loro leader storici (Demirel, Ecevit, Erbakan) ${ }^{8}$. L'intento della giunta militare fu di attuare cambiamenti radicali all'intero sistema politico turco9 ${ }^{9}$ Altrettanto decisiva, quindi, risultò la legittimazione ricevuta da Özal per mano della giunta militare, che lo considerava un politico conservatore, le cui idee avrebbero rafforzato i principi alla base del nazionalismo turco-kemalista.

Un ulteriore fattore decisivo fu la figura stessa di Özal. Egli era un uomo d'affari, fervente ammiratore degli Stati Uniti - dove aveva studiato - e del liberalismo, a cui abbinava una formazione politica fortemente religiosa (era stato candidato del partito islamico) ${ }^{10}$. L'immagine politica di Turgut Özal rappresentò un elemento di novità: si presentò con tratti considerati ambivalenti in un contesto, come quello turco, in cui si era soliti considerare un esponente politico in quanto modernista laico oppure tradizionalista religioso.

Altrettanto importante, infine, fu il modo di porsi di Özal nei confronti della giunta militare, che lo considerava come un fattore fondamentale ai fini della ripresa economica. La collaborazione con loro avrebbe infatti garantito la stabilità del Paese, necessaria per attuare le riforme ritenute fondamentali per la ripresa dell'economia e per sbloccare nuovi fondi provenienti dal Fondo Monetario Internazionale ${ }^{11}$.

L'ANAP vinse le elezioni (45,15\%) presentandosi come una sintesi dei quattro principali orientamenti politici turchi degli anni Settanta (destra, sinistra, integralismo

7 ZURCHER, Erik Jan, Turkey: A Modern History, London, I. B. Tauris, 1993, p. 324.

${ }^{8}$ Ibidem, p. 342.

9 Vennero sciolti anche tutti i Consigli Municipali, circa 1.700, e destituiti tutti i sindaci.

10 NOCERA Lea, La Turchia contemporanea. Dalla repubblica kemalista al governo dell'Akp, Roma, Carocci, 2011, p. 69.

${ }^{11}$ Il FMI, così come OCSE e la Banca mondiale, vincolarono il prestito alla Turchia $(1,8$ miliardi di dollari) all'introduzione di una serie di riforme economiche, finanziarie e commerciali. 
islamico, destra radicale). Queste forze, tenute insieme dall'energica personalità di Özal, ottennero consensi e seguito soprattutto presso le classi medie, che avrebbero goduto delle politiche economiche messe in atto dal nuovo governo ${ }^{12}$.

A godere maggiormente delle riforme, infatti, fu una nuova classe media non legata direttamente ad Istanbul ma piuttosto alle province anatoliche e, come tale, meno vicina all'establishment kemalista ma più alla tradizione religiosa e mistica delle confraternite sufi a cui sia Özal che Erdoğan appartenevano ${ }^{13}$. Partendo da questi presupposti si mise in moto un meccanismo che risulterà decisivo nei primi anni del secondo millennio per l'ascesa dell'AKP e la definitiva affermazione dell'Islam politico.

Le aperture liberali volute da Özal e la graduale rivalutazione del valore islamico della società turca promossa dalla giunta militare, che la considerò un valido argine alle derive rivoluzionarie dei movimenti di sinistra ${ }^{14}$, contribuirono ad incentivare l'elaborazione di una cultura autonoma rispetto al Kemalismo e una sua progressiva politicizzazione ${ }^{15}$.

Formulata da un gruppo di intellettuali e professori universitari, conosciuti come Aydınlar Ocağ $1^{16}$, la sintesi turco-islamica verteva sull'idea che la cultura islamica fosse un complemento morale necessario al rafforzamento dei valori d'ordine incarnati nel nazionalismo turco ${ }^{17}$. Elemento caratteristico della politica della giunta militare, infatti, fu l'adozione come parte integrante della propria ideologia della sintesi turco-islamica (Türk-İslam Sentezi), la quale contribuì a temperare il pensiero islamista e a tessere uno stretto legame tra i militari e l'ANAP guidato da Özal ${ }^{18}$.

Le riforme economiche - contrassegnate dell'apertura al mercato globale -, portarono sulla scena culturale turca una nuova classe di moderni intellettuali islamici. Questi, provenienti dalla borghesia religiosa, introdussero nel contesto turco pratiche di consumo e comunicazione tipiche dell'Occidente, adattandovi il proprio messaggio

\footnotetext{
${ }_{12}$ OZZANO, Luca, «L'AKP: islamocrazia come modello?», in Aspenia, 16, 52, 2011, pp. 136-142. ${ }^{13}$ Sull'importanza degli ordini sufi si veda: YAVUZ, Hakan, Islamic Political Identity in Turkey, New York, Oxford University Press, 2003, pp. 133-145.

${ }_{14}$ Un processo simile venne intrapreso negli anni settanta in Egitto da Sadat che favorì il diffondersi delle Gama'at al-Islamiyya (Associazioni Islamiche) al fine di arrestare lo sviluppo delle forze rivoluzionarie di sinistra, sull'argomento si veda: KEPEL, Gilles, Jihad: expansion et déclin de l'islamisme, Paris, Gallimard, 2000, pp. 87-105.

${ }^{15}$ YAVUZ, Hakan, Islamic Political Identity in Turkey, cit., p. 217.

16 "Focolare degli intellettuali".

${ }^{17}$ KURT, Umit, «The Doctrine of Turkish-Islamic Synthesis as official Ideology of the September 12 and the Intellectuals Hearth as the Ideological Apparatus of the State», in European Journal of Economic and Political Studies, 2, 2010, pp. 111-125.

18 TOPRAK, Binnaz, Religion as State Ideology in a Secular Setting: The Turkish-Islamic Synthesis, in WAGSTAFF, Malcolm (ed.), Aspects of Religion in a secular Turkey, Center for Middle Eastern Studies and Islamic Studies, University of Durham UK, 1990, pp. 10-14.
} 
culturale e religioso ${ }^{19}$. Iniziò, quindi, ad essere eroso quel principio di laicità (laiklik), voluto da Kemal Atatürk ed ispirato alla laïcité francese, in cui il secolarismo appariva ancora ad uno stadio ibrido, ossia dove elementi rintracciabili nell'anticlericalismo francese si coniugavano ad influenze direttamente riconducibili all'Impero ottomano ${ }^{20}$. In particolare, rispetto al laicismo francese, in cui lo Stato rimane neutro e si disinteressa delle vicende religiose, la Repubblica kemalista, come già avvenuto durante gli ultimi anni di vita dell'Impero, puntò a controllare la sfera religiosa attraverso l’istituzione del Diyanet Isşleri Müdürlüğ ̈̈²1.

L' Islam così acquisì nuovi spazi di espressione, cessando di essere rinchiuso nella sola sfera del privato ed assumendo una valenza identitaria collettiva, che non rinnega la modernità ma sperimenta e professa un nuovo modo di vivere, distante da quello kemalista, in cui centrali sono l'etica islamica e il recupero del proprio passato ottomano a lungo rinnegato.

\section{L'ottomanismo alla base della nuova identità turca}

Per portare avanti le proprie politiche, Özal avvertì subito la necessità di una nuova definizione dell'identità turca che andasse a sostituire quella kemalista, ormai non più adatta a interpretare i cambiamenti sociali e globali del Paese.

Per Kemal l’identità turca era definita dalla koinè linguistica e territoriale rigettando, sotto l'influenza del modello francese, il multi-culturalismo che aveva rappresentato uno dei principali pilastri dell'Impero ottomano.

Il forte nazionalismo assimilazionista promosso dal Kemalismo (Atatürkçülük), tuttavia, aveva portato a discriminazioni etniche maggiori di quelle possibili sotto l'Impero ottomano, dove la coabitazione culturale, confessionale ed etnica rappresentò per secoli un carattere peculiare ${ }^{22}$. L'impostazione kemalista, quindi, portò alla rottura

19 YAVUZ, Hakan, Islamic Political Identity in Turkey, cit., p. 112.

20 TASPINAR, Ömer, «Turkey's Middle East Policies: between Neo-Ottomanism and Kemalism», in Carnegie Papers, 10, 2008, pp. 4-5.

${ }_{21}$ "Direzione per gli Affari religiosi". Il Diyanet è un organo governativo tuttora esistente che ha tra le sue principali funzioni quella di dettare le linee guida dell'Islam turco: questo avviene attraverso la definizione dei programmi educativi delle scuole per predicatori (imam), oltre checon il coordinamento e la gestione di tutte le questioni burocratiche e finanziarie dell'apparato religioso. Sull'argomento si veda: KEPEL, Gilles, Jihad: expansion et déclin de l'islamisme, cit., p. 49. Sullo sviluppo del concetto di laicità nella Turchia moderna si consiglia: DOGAN, Ali Kemal, «L'AKP et l'évolution de la laïcité en Turquie», in Moyen-Orient, n. 9, 2011, pp. 36-41.

${ }_{22}$ TASPINAR, Ömer, «Turkey's Middle East Policies: between Neo-Ottomanism and Kemalism», cit., p. 5 . 
con la comunità curda; rottura che provocherà una delle più delicate e sanguinose questioni interne della politica repubblicana ${ }^{23}$.

Özal era conscio che la Turchia necessitasse un nuovo riconoscimento (dal punto di vista costituzionale e pubblico) delle molte realtà presenti all'interno del Paese. I diversi caratteri etnici, culturali, religiosi, linguistici e politici erano considerati da Özal come valori da comprendere in una nuova identità nazionale ${ }^{24}$, in grado di assumere un carattere inclusivo più ottomano che turco. Un'identità che avrebbe dovuto ricalcare alcuni dei principali tratti di quella elaborata nel XIX secolo, durante la stagione delle riforme Tanzimat (1839-1876)25, dall'élite burocratica a capo del processo di modernizzazione dell'Impero: l'ottomanismo (in turco Osmanlilik) ${ }^{26}$.

Tale ideologia derivava da uno dei principi cardine del processo di Tanzimat, ovvero l'idea che tutti i cittadini dovessero godere dello stesso status all'interno dell'Impero. Nonostante questo fosse un concetto puramente formale, che trovò piena applicazione nella legge sulla cittadinanza ottomana approvata nel 1869, una volta sommato alle riforme istituzionali portate avanti in quegli anni, iniziò a costituire una prima embrionale elaborazione identitaria che prescindeva dalle singole appartenenze religiose ed etniche.

Paradossalmente fu proprio questo fallito tentativo di dare vita ad un moderno concetto di cittadinanza che, ponendo i sudditi dell'Impero sullo stesso piano - senza più distinzioni etniche e confessionali -, si rivelò essere uno dei principali fattori che determinarono il crollo ottomano.

L'ottomanismo, infatti, intaccò il sistema amministrativo e organizzativo dei millet, che fino a quel momento avevano rappresentato la migliore espressione della tolleranza religiosa ottomana, figlia della "pragmatica" convinzione che l'accettazione delle differenze (etniche e religiose) fosse necessaria per la sopravvivenza di una realtà pluriconfessionale come l'Impero ${ }^{27}$.

23 BOZARSLAN, Hamit, La Turchia contemporanea, Bologna, Il Mulino, 2006, pp. 84-87.

24 LAÇINER, Sedat, "Turgut Özal period in Turkish Foreign Policy: Ozalism», in USAK Yearbook of International Politics and Law, 2, 2009, pp. 153-205 URL:

$<\quad$ http://www.turkishweekly.net/article/333/turgut-ozal-period-in-turkish-foreign-policyozalism.html $>$ [consultato il 2 febbraio 2012].

25 Sull'argomento si veda: MANSFIELD, Peter, A History of Middle East, London, Penguin, 1991, pp. 71-95; CAMPANINI, Massimo, Storia del Medio Oriente, Bologna, Il Mulino, 2006, pp. 25-29.

${ }_{26}$ DEMİAG, Yelda, «Pan-Ideologies in the Ottoman Empire against the West: From PanOttomanism to Pan-Turkism», in The Turkish Yearbook of International Relations, 36, 2005, pp. 139-156.

27 DEL ZANNA, Giorgio, I cristiani e il Medio Oriente (1798-1924), Bologna, Il Mulino, 2011, pp. 78-79. Si veda anche: ZARCONE, Thierry, La Turquie moderne et l'Islam, Paris, Flammarion, 2004 . 
A crollare, prima ancora che l'Impero, fu la coabitazione, intesa come convivenza pacifica di una molteplicità di identità, che per secoli aveva costituito l'asse portante della società ottomana.

Una più attenta analisi dell'ottomanismo evidenzia come sia possibile riscontrare una sua evoluzione in tre differenti stadi tra il (1856) e il (1882), inevitabilmente legati e influenzati da precise contingenze storiche ma, allo stesso tempo, accomunati dalla volontà di arrestare il declino dell'Impero.

La prima fase (1856) definibile come ottomanismo "liberale" fu caratterizzata dalle idee di diversi esponenti della Sublime Porta, su tutti i Gran Vizir Alì Pascià e Fuad Pascià, volte alla promozione di una nuova idea di cittadinanza come unione delle diverse etnie dell'Impero (Ittihad-i Ansar).

La seconda fase, invece, vide protagoniste le idee elaborate e promosse dal gruppo di intellettuali riformatori conosciuti come Giovani Ottomani (1865-1878). Essi furono i primi a tentare una mediazione tra le idee di stampo europeo (liberalismo, costituzionalismo) con la specificità islamica dell'Impero e della sua popolazione e coniarono un'idea di unione di elementi ispirata alla comunità islamica (Ittihad-i Islam) che consideravano nella sua forma originaria come un embrione di democrazia ${ }^{28}$.

La terza fase dell'ottomanesimo, infine, fu quella delineata dal Sultano Abdülhamit II (1876-1909) il quale, a causa delle ingenti perdite subite in termini territoriali e di popolazione nel 1878 a seguito del Trattato di Berlino, accentuò il carattere islamico dell'Impero e della sua autorità ${ }^{29}$. Il Sultano, infatti, per legittimare il proprio ruolo diede vita ad una forma di pan-islamismo in chiave difensiva, volto principalmente a compattare la comunità ottomana intorno ai simboli della tradizione islamica (Califfo, califfato) ${ }^{30}$.

Nonostante i suoi sviluppi l'ottomanismo fallì nel suo compito più arduo ovvero il contrasto delle molte forze centrifughe interne che indebolirono l'Impero ottomano rendendolo facile preda delle mire imperialiste europee.

Il richiamo di Özal all'ottomanismo, oltre all'aspetto identitario, si legava anche alla propria ammirazione nei confronti degli Stati Uniti, che considerava un modello ideale per la moderna Turchia.

${ }^{28}$ Sull'argomento si veda: MARDIN, Serif, The Genesis of Young Ottoman Thought: A Study in the Modernization of Turkish Political Ideas, Princeton CA, Princeton University Press, 1962.

${ }^{29}$ ZURCHER, Erik Jan, Turkey: A Modern History, cit., pp. 96-97.

3o Per un'attenta analisi sul mutamento simbolico ed iconografico che interessò la figura del Sultano si veda: DERINGIL, Selim, The Well-Protected Domains: Ideology and the Legitimation of Power in the Ottoman Empire 1876-1909, London, I. B. Tauris, 1999, pp. 15-43. 
Özal riteneva che i due Paesi avessero avuto in passato una struttura politica molto simile, che aveva consentito a culture differenti di coesistere in modo libero e pacifico salvaguardando le proprie preferenze religiose.

Un altro elemento - che non poteva più essere sottovalutato dal governo turco - era la presenza di un gran numero di migranti provenienti da regioni vicine (Medio Oriente) e lontane (Asia Centrale), riproposizione di quanto già successo nell'Impero ottomano. La rilevanza della Turchia in quanto polo attrattivo per le popolazioni dell'area, infatti, aveva portato all'annessione di varie etnie profondamente diverse tra loro. Le molte etnie furono assimilate con la forza all'identità turca poiché gli fu impedita qualsiasi forma di rappresentanza politica e comunitaria. Ciò non impedì la loro crescita progressiva, che trovò negli anni Ottanta sbocco in vari gruppi di pressione la cui influenza non poteva essere ignorata ancora a lungo.

Özal si accorse di questo ulteriore importante cambiamento maturando la convinzione che le esigenze delle molteplici etnie e dei relativi gruppi di pressione dovessero trovare risposte nelle politiche del Paese. Una considerazione che diventerà determinante nella nuova linea di politica estera impostata da Özal, tendente al recupero sempre maggiore delle relazioni con i Paesi vicini e le ex-province ottomane ${ }^{31}$. La Turchia non poteva più disinteressarsi di ciò che stava avvenendo oltre i propri confini: una politica più attiva era espressamente richiesta dai molti gruppi di pressione etnici.

L’idea di società "turca" di Özal era molto diversa da quella professata da Atatürk il quale considerava come possibile minaccia tutto ciò che era esterno all'Anatolia ${ }^{32}$. Il "turchismo" di Özal era in realtà un concetto fortemente culturale, che non intendeva scontrarsi con le etnie minoritarie del Paese, ma mirava piuttosto ad un loro assorbimento nel più ampio concetto di "turchi" o, più precisamente, sotto quello di ottomani. Se Kemal aveva sognato una società secolarizzata e occidentalizzata con un suo posto in Europa, Özal voleva dar vita ad una società democratica, musulmana, liberale e capitalista racchiusa all'interno di un sistema statale che fosse multiculturale, multilinguistico e multireligioso ${ }^{33}$.

${ }^{31}$ LAÇİNER, Sedat, «Turgut Özal period in Turkish Foreign Policy: Ozalism», cit., pp. pp. 153205 .

32 Ibidem.

33 TASPINAR, Ömer, «Turkey's Middle East Policies: between Neo-Ottomanism and Kemalism», cit., p. 11. 


\section{L'ozalismo e il riavvicinamento alle province ottomane}

Il neo-ottomanesimo - definito in questa fase "ozalismo" - emerse nella politica estera turca come decisa reazione alla politica isolazionista voluta dal Kemalismo e dalla ferma convinzione che la Repubblica di Turchia potesse tornare a svolgere un importante ruolo di potenza sia a livello regionale che globale. Un'impostazione che per Özal fu anche un obbligo dettato dall'isolamento e dalle pessime condizioni economiche in cui versava la Turchia. I primi anni sotto la sua guida, pertanto, furono caratterizzati dal tentativo di reinserire la Turchia nel contesto internazionale, viste le tensioni sia con l'Europa che con gli Stati Uniti34.

La politica estera di Özal divenne sempre più funzionale alla protezione degli interessi economici turchi nel mondo; se prima le relazioni internazionali seguivano logiche strategiche, con Özal iniziarono ad essere determinate dagli interessi economici35. Özal cercò lentamente di recuperare i rapporti sia con gli Stati Uniti che con l'Europa, ma allo stesso tempo iniziò a considerare nuovi partner aprendo il Paese a relazioni sempre maggiori con gli altri Stati della regione. Si verificò un deciso incremento nei rapporti con i Paesi dei Balcani, del Mar Nero e soprattutto del Medio Oriente, anche in virtù dell'aumento della domanda di petrolio turca, dovuta alla rapida ripresa economica ${ }^{36}$.

Un altro segnale forte del mutato approccio turco fu il cambio di politica sulla questione israelo-palestinese con l'appoggio sempre maggiore fornito all'OLP37, che per anni era stato osteggiato a causa dei suoi legami con il gruppo di guerriglia armeno ASALA.

Nella nuova politica estera turca, tuttavia, giocarono un ruolo decisivo anche fattori esterni, determinati da quel continuo intreccio tra globale e regionale che caratterizzò gli anni della guerra fredda: su tutti la rivoluzione iraniana e l'ingresso delle truppe sovietiche in Afghanistan, che portarono ad un nuovo avvicinamento con gli Stati

\footnotetext{
34 NOCERA, Lea, La Turchia contemporanea, cit.

35 LAÇiNER, Sedat, «Turgut Özal period in Turkish Foreign Policy: Ozalism», cit.

${ }^{36} \mathrm{La}$ bilancia commerciale, quindi, necessitava di trovare un equilibrio tra import ed export e questo portò all'apertura degli scambi e degli investimenti turchi in nuovi Paesi come la Libia, che divenne il principale mercato turco, l'Arabia Saudita oppure l'Iraq di Saddam Hussein. Si veda: TASPINAR, Omer, «Turkey's Middle East Policies: between Neo-Ottomanism and Kemalism», cit., p. 11.

37 La prima sede in Turchia venne ufficialmente aperta nel 1976.
} 
Uniti $3^{8}$. A differenza del Kemalismo che la considerava come una possibile minaccia, Özal vedeva l'avvicinamento e l'integrazione all'Occidente come funzionale alla sicurezza, anche interna, della Turchia. Per questo motivo, le azioni in politica estera intraprese dalla Turchia furono sempre più legate alle scelte di Washington ${ }^{39}$.

Negli anni successivi Ankara dimostrò una grande capacità di sfruttare a proprio vantaggio i molti cambiamenti degli assetti geopolitici regionali e globali; ne fu un esempio la capacità, nei primi anni Novanta, di crearsi un proprio spazio di manovra, comunque su incoraggiamento degli Stati Uniti, nelle ex-Repubbliche sovietiche dell'Asia Centrale, approfittando della contrazione territoriale e politica della Russia. Il crollo dell'Unione Sovietica e la nascita di cinque repubbliche "turcomanne"40 suscitarono reazioni euforiche presso l'opinione pubblica turca, tanto che Özal salutò l'evento dichiarando la nascita di un «nuovo secolo turcomanno»41.

Özal inserì un altro importante elemento di novità nella politica estera turca: l'aspetto culturale, che ha sempre giocato nell'ideologia neo-ottomana un ruolo di primo piano e che spinse Özal prima e l'AKP poi, ad avvicinarsi sempre più ai territori delle ex-province ottomane.

Anticipando quella che sarebbe stata una prerogativa della politica estera di Davutoğlu, Özal introdusse nelle relazioni con le ex-province ottomane il sempre maggiore utilizzo del "soft power"; tuttavia, a differenza di quanto sarà poi fatto dall'AKP, Özal non poté godere di ampi margini di manovra a causa di un'economia non ancora abbastanza forte e affidabile.

Özal, quindi, ebbe il merito di emancipare concetti come "islamismo" e "ottomanismo", ritenuti per anni dai kemalisti pericolosi per la Turchia, facendoli diventare valori e punti di contatto su cui costruire nuove relazioni. Argomenti che saranno successivamente fatti propri e approfonditi dall'AKP4².

\footnotetext{
${ }^{38}$ HALLIDAY, Fred, The Middle East in International Relations. Power, Politics and Ideology, Cambridge, Cambridge University Press, 2005.

39 Ibidem.

40 Azerbaijan, Turkmenistan, Uzbekistan, Kazakistan e Kirghizistan. La Turchia fu il primo Paese a riconoscerle a livello diplomatico.

${ }^{41}$ ZURCHER, Erik Jan, Turkey: A Modern History, cit., p. 397.

${ }^{42}$ NOCERA, Lea, La Turchia contemporanea, cit.; TASPINAR, Ömer, «Turkey's Middle East

Policies: between Neo-Ottomanism and Kemalism», cit., pp. 14-16.
} 


\section{La vittoria dell'AKP e dell'islamismo moderato}

L'avvento al potere dell'Adalet ve Kalkınma Partisi (AKP) 43 alle elezioni del novembre 2002, ha rappresentato il decisivo punto di rottura della politica turca rispetto al passato.

La specificità del caso turco risiede proprio nella capacità di produrre un partito di governo che pur affondando le proprie radici nella tradizione islamica - il movimento Milli Gorus ${ }^{44}$ di Necmettin Erbakanh - ha saputo coniugare l'Islam al libero mercato e a cospicue aperture nei confronti dell'Occidente, in particolare dell'Unione Europea.

Solamente dando vita ad un movimento politico più maturo e meno vulnerabile agli attacchi dei militari e della magistratura, si sarebbe potuto rafforzare il ruolo politico dell'Islam all'interno delle strutture di una democrazia laica quale quella turca45.

Erdoğan e Gül, infatti, partendo dall'esperienza di governo di Erbakan tra il 1995 e il 1997, compresero l'importanza di dare vita ad una formazione politica ibrida in cui le radici islamiche si incontravano con i tradizionali valori del nazionalismo e con la ferma ambizione di avvicinarsi ulteriormente al mondo occidentale ed in particolare all'Unione Europea.

La capacità dei due leader del AKP, l'attuale Presidente Gül e il Premier Erdoğan, è stata quella di sancire uno strappo non solo con il Kemalismo ma anche con il vecchio islamismo politico attraverso un rapido processo di maturazione e temperamento, favorito da dinamiche endogene ed esogene.

Erdoğan, tuttavia, ebbe il merito e il coraggio di richiamarsi ad un passato politico considerato scomodo dalla giunta militare e dall'élite kemalista. Egli fu, infatti, il primo ed unico leader politico turco ad identificarsi con lo spirito politico che avevano avuto in precedenza Ertekin Menderes ${ }^{46}$ - Primo Ministro negli anni Cinquanta - e Özal negli anni Ottanta. Come loro, anche lui ereditava un Paese in profonda crisi, economica e politica e, come loro, si proponeva di guidarlo attraverso una difficile transizione verso nuovi equilibri democratico istituzionali.

43 "Partito della Giustizia e dello Sviluppo".

44 "Vista Nazionale".

45 ZURCHER, Erik Jan, Turkey: A Modern History, cit., pp. 370-371. Sull'argomento si veda: HALE, William, ÖZBUDUN, Ergun, Islamism, Democracy and Liberalism in Turkey: The Case of the Akp, London, Routledge Studies in Middle Eastern Politics, 2010.

46 Primo Ministro dal 1950 al colpo di Stato del 1960; dopo essere stato deposto dai militari, venne giustiziato con l'accusa di attentato alla Costituzione turca. 
Risulta quindi corretto considerare la vittoria dell'AKP nel 2002 non come un "terremoto politico" ma, piuttosto, come un processo avviato dalla volontà di restaurare qualcosa che già era esistito in passato 47 .

A ciò l'AKP ha saputo però conciliare caratteri moderni, presentandosi come piattaforma "democratica conservatrice" 48 in grado di rispondere pienamente alle esigenze del nuovo ceto imprenditoriale sviluppatosi nelle province anatoliche grazie alle politiche economiche avviate dal governo Özal, le cosiddette Tigri anatoliche. Una nuova borghesia che vede nell'Europa e nella democrazia non frutti avvelenati della colonizzazione e dell'imperialismo occidentale, bensì occasione per l'acquisizione di maggiori libertà in diversi ambiti, compreso quello religioso ${ }^{49}$.

L'ascesa di un partito di chiara origine islamica, per quanto moderato, ha inoltre contribuito all'erosione del potere detenuto dai militari. L'esercito, in Turchia, ha legittimato fin dal primo golpe repubblicano (1960) il proprio ruolo di garante ed interprete dei valori e dei principi kemalisti, giustificando e rafforzando tale ruolo ipotizzando possibili minacce interne al Paese.

Un ruolo, quello dei militari, riconosciuto costituzionalmente nella veste del Consiglio di Sicurezza nazionale, organo costituzionale anomalo con a capo i vertici delle forze armate, ufficialmente incaricato di occuparsi della sicurezza nazionale ${ }^{50}$.

Per anni i principali pericoli all'unità nazionale sono stati identificati con le rivendicazioni separatiste curde e con l'Islam politico in quanto promotore di uno Stato islamico.

La progressiva riabilitazione dell'Islam nella sfera pubblica e in quella politica turca ha gradualmente disinnescato la minaccia rappresentata dall'islamismo radicale e, al tempo stesso, promosso una possibile, seppur improbabile, soluzione alla questione curda, rappresentata dall'idea di identità multietnica che integra, invece di assimilare come quella kemalista, le diversità etniche.

Questi sviluppi hanno segnato il progressivo, seppur lento, calo di popolarità dell'esercito, accompagnato da una generale disaffezione popolare nei confronti dello stesso mito kemalista. Una disaffezione seguita da due pesanti sconfitte politiche incassate dai militari.

\footnotetext{
47 YAVUZ, Hakan, Islamic Political Identity in Turkey, cit., p. 257.

48 Sull'argomento si veda: HALE, William, ÖZBUDUN, Ergun, Islamism, Democracy and Liberalism in Turkey: The Case of the Akp, cit., p. 24; NOCERA, Lea, La Turchia contemporanea, cit., p. 104.; YAVUZ, Hakan, Islamic Political Identity in Turkey, cit., pp. 260261.

49 OZZANO, Luca, «Turkey's Middle East Policies: between Neo-Ottomanism and Kemalism», cit., pp. 137-138.

50 BERNARDINI D'ARNESANO, Beatrice, CARDUCCI, Michele, Turchia, cit., pp. 66-67.
} 
La prima è avvenuta con l'ascesa del AKP al ruolo di partito di governo: il risultato più eclatante è rappresentato dall'elezione alla carica di Presidente di Abdullah Gül, primo Presidente islamico della Repubblica. La seconda si è verificata con il fallimento del "comunicato di mezzanotte" del 2007; i militari sperimentarono un metodo comunicativo diverso, non più una diretta minaccia al governo - come avvenuto nel febbraio del 1997 nei confronti del governo a guida Erbakan - ma, piuttosto, un invito a mobilitare l'opinione pubblica facendo un ampio uso del mezzo di comunicazione più diretto e moderno: il web.

Il comunicato di mezzanotte risultò l'ultimo tentativo da parte dell'esercito di intromettersi negli affari politici ed istituzionali del Paese ${ }^{51}$, segnando anche una decisiva svolta nelle politiche promosse dall'AKP che, a partire da quello stesso anno, acquisirono un carattere più marcatamente islamico ${ }^{52}$.

Gli effetti principali di questo mutamento di prospettiva si sono avuti in politica estera, con il progressivo allontanamento da partner storici come l'Unione Europea e Israele, e in politica interna, dove, attraverso il processo di introduzione della propria élite nei diversi apparati amministrativi statali (educazione, economia, informazione, affari religiosi), l'AKP ha potuto portare avanti la trasformazione dell'intera società civile turca.

Nei suoi primi anni di governo, invece, l'AKP ha saputo utilizzare la spinta, anche popolare, all'adesione all'Unione Europea sia per fugare dubbi e timori occidentali di derive islamiche, sia per accelerare le riforme interne al Paese. Proprio le misure conosciute come "pacchetti di armonizzazione" rispetto alle norme comunitarie hanno liberalizzato ulteriormente l'economia, andando ad intaccare il controllo diretto statale e la politica, limitando ulteriormente l'influenza dei militari53.

Il voto elettorale in favore dell'AKP, pertanto, deve essere letto come volontà da parte della società civile di legittimare un nuovo attore avente mandato "implicito" di ristrutturare il confine tra Stato e società 54 .

Le decisioni prese in questi anni dall'AKP sono confortate da un largo consenso da parte di una popolazione a cui non sembra vero di essere riuscita a trovare in un decennio la definitiva stabilità politica oltre ad una impressionante ed inaspettata

51 BERNARDINI D'ARNESANO, Beatrice, CARDUCCI, Michele, Turchia, cit., pp. 83-85; NOCERA, Lea, La Turchia contemporanea, cit., pp. 106-108.

$5^{2}$ DOGAN, Ali Kemal, «L'AKP et l'évolution de la laïcité en Turquie», cit.

53 OZZANO, Luca, «Turkey's Middle East Policies: between Neo-Ottomanism and Kemalism», cit., p. 138. Per una valutazione completa delle riforme volte all'armonizzazione con l'Unione Europea si veda: BERNARDINI D'ARNESANO, Beatrice, CARDUCCI, Michele, Turchia, cit., pp. 135-149.

54 YAVUZ, Hakan, Islamic Political Identity in Turkey, cit., p. 257. 
crescita economica (9,2\% nel 2011) che ha portato la Turchia al sedicesimo posto tra le economie mondiali.

Va quindi evidenziato come il fattore decisivo per il successo del disegno politico proposto dall'AKP sia stato il contesto globale. In particolare i trend economici dell'ultimo decennio si sono dimostrati favorevoli ai Paesi in via di sviluppo, che nel giro di pochi anni hanno saputo lanciarsi nel mercato globale.

Il successo in ambito economico ha aiutato a mantenere gli ottimi rapporti con la borghesia economica e religiosa ma ha anche permesso allo Stato una lunga serie di interventi volti al miglioramento delle condizioni di vita delle fasce più povere della società, disinnescando elementi potenzialmente destabilizzanti per l'equilibrio sociale turco.

\section{Il richiamo al passato ottomano della "profondità strategica" di Davutoğlu}

La posizione della Turchia durante gli anni di guerra fredda era comunemente associata a quella di un Paese di frontiera, nozione che è cominciata a mutare nel 1990 con la fine del mondo bipolare ed è divenuta quella attuale di Paese ponte55. Una definizione che, nonostante appaia troppo abusata, rispecchia bene il ruolo di collegamento tra Occidente e Oriente svolto dalla Turchia e che richiama il ruolo in passato ricoperto dall'Impero ottomano.

Con l'ascesa dell'AKP, la centralità geopolitica della Turchia ha iniziato a svilupparsi definendo nuovi indirizzi in politica estera, caratterizzati da una crescente e legittima autonomia. Determinante è stata l'influenza di Ahmet Davutoğlu, ministro degli esteri dal 2009 ma da sempre vicino ad Erdoğan, e della sua dottrina della "profondità strategica" 56 .

Una dottrina, incentrata sulla retorica dell'“incontro tra civiltà”, che rientra nel più ampio e complesso quadro del neo-ottomanesimo e mira a trasformare la Turchia da Stato periferico ad attore centrale nel contesto regionale prima e in quello globale poi 57.

55 DAVUTOGLU, Ahmet, «Turkey's New Foreign Policy Vision», in Insight Turkey, 10, 1/2008, pp. 77-96.

${ }^{6}$ Il testo di Davutoğlu è DAVUTOGLU, Ahmet, Stratejik derinlik: Türkiye'nin uluslararası konumu, Istanbul, Küre Yayınlari, 2001. Parti tradotte del testo si trovano in: DAVUTOGLU, Ahmet, «Profondità Strategica. Il mondo secondo Ankara», in Limes 4/2010, pp. 29-39.

57 TASPINAR, Ömer, «Turkey's Middle East Policies: between Neo-Ottomanism and Kemalism», cit., pp. 14-17. 
A prevalere è l'idea di una Turchia in grado di mediare tra i due mondi, Occidente ed Islam, diventandone l'anello di congiunzione ${ }^{5}$.

L'asset fondamentale nella dottrina di Davutoğlu, attuale e più completa realizzazione del neo-ottomanesimo59, risulta essere il progressivo recupero dell'eredità ottomano islamica. In questo approccio, centrale è l'idea dell'AKP dell'Islam come fattore non più destabilizzante, come riteneva invece il Kemalismo, bensì aggregante. Al richiamo all'eredità islamica, il governo turco aggiunge una politica estera che, rifacendosi a quella intrapresa da Özal, riflette un maggiore attivismo negli ex-territori dell'Impero in ambito politico, economico e culturale. Un disegno che non è volto a perseguire una nuova fase di imperialismo, quanto piuttosto a un sempre più diffuso uso di soft power nelle ex province ottomane (Balcani, Medio Oriente, Asia Centrale). In quest'ottica si deve comprendere l'enunciazione, dello stesso Davutoğlu, del principio di "zero problemi con i vicini", che ha portato a sviluppi decisivi nella normalizzazione delle relazioni con Paesi come Armenia e Grecia e, in parte, anche con Cipro. La politica di zero problemi si basa essenzialmente sulla progressiva eliminazione di relazioni impostate in maniera conflittuale con i Paesi confinanti e più in generale con tutti quelli adiacenti alla regione, attraverso la risoluzione pacifica delle controversie e dei possibili fattori di tensione.

La peculiarità della retorica "zero problemi" risiede nel suo forte carattere transnazionale, emerso in tutta la sua forza durante i mesi successivi alle rivolte arabe, che ha consentito alla Turchia di creare relazioni soprattutto con i popoli vicini più che con gli Stati. La tendenza è stata quella di ramificare le relazioni in più ambiti, soprattutto economico e culturale, spesso scavalcando i regimi e le istituzioni, ed andando a creare legami direttamente con la popolazione ${ }^{60}$.

Questa visione comporta implicazioni anche interne con una revisione, come già compreso da Özal, del concetto di identità nazionale. Il neo-ottomanesimo comporta l'apertura alle molte minoranze etniche del Paese attraverso l'elaborazione di un concetto multi-culturale di cittadinanza che avrà inevitabili conseguenze nel rapporto con i curdi ${ }^{61}$. Un concetto che non solo si rifà alle idee di Özal in materia, ma richiama

58 HEPER, Metin, «The Justice and Development Party Government and the Military in Turkey», in Turkish Studies, 6, n.2/2005, pp. 220-229.

59 Sull'argomento si veda: BOZDAGLIOGLU, Yücel, «Modernity, Identity and Turkey’s Foreign Policy», in Insight Turkey, 10, 1/2008; DAVUTOGLU, Ahmet, «Turkey's New Foreign Policy Vision», cit.

6o AKYOL, Mustafa, “Turkey’s maturing foreign policy”, in Foreign Affairs July/2011. URL:

< http://www.foreignaffairs.com/articles/67974/mustafa-akyol/turkeys-maturing-foreignpolicy $>$ [Consultato il 15 febbraio 2012].

61 TASPINAR, Ömer, «Turkey's Middle East Policies: between Neo-Ottomanism and Kemalism», cit., p. 15 . 
direttamente l'ideologia dell'ottomanismo e la sua idea di "unità di elementi" sotto una veste ancora più specifica. L'AKP accentua la presenza di un forte richiamo all'identità islamica riprendendo l'ottomanismo nella sua terza fase, ovvero quella del periodo hamidiano. In questo periodo, come già evidenziato in precedenza, l'idea di ottomanismo venne rivestita dal Sultano Abdülhamit II di un simbolismo di matrice islamica, volto a dare nuova e maggiore legittimità al proprio potere ${ }^{62}$. Una scelta politica e strumentale del Sultano, che portò alla ripresa del titolo di Califfo, che però non presentava il carattere espansionistico tipico del panislamismo moderno, ma, si prefissava l'intento di rafforzare la coesione interna all'Impero ${ }^{63}$. Anche per l'AKP l'Islam può e deve essere la chiave e l'amalgama per una nuova identità sempre più sentita e condivisa dalle diverse etnie. A ciò si deve aggiungere come Davutoğlu ed Erdoğan - così come prima di loro Özal e lo stesso Sultano Abdülhamit II condividessero la visione di un Islam dinamico e progressista perfettamente in grado di adattarsi ed esprimersi nella modernità. Un'idea figlia della forte influenza avuta su tutti loro dalla confraternita sufi Nakşibendi, di cui sia Erdoğan che Özal sono stati membri.

Quella professata da Davutoğlu ed Erdoğan, quindi, non è in alcun modo un islamizzazione ma, piuttosto, una sorta di riconciliazione con l'Islam e, al tempo stesso, correzione dell'approccio laico del Kemalismo ${ }^{64}$.

Argomenti questi che, al pari del successo economico degli ultimi anni, hanno fatto breccia nel mondo musulmano facendo crescere l'ammirazione verso il modello turco e il suo leader Erdoğan. Per capitalizzare questa ammirazione il governo dell'AKP ha dato vita ad una politica regionale sempre più attiva, trovando nell'Islam l'elemento comune da cui partire. In quest'ottica si spiega l'importanza del recupero del discorso islamico in un contesto, come quello dei Balcani65 e del Medio Oriente, in cui l'appartenenza comunitaria e confessionale ha ancora un ruolo primario e determinante nelle relazioni tra Stati e tra popoli.

Questo approccio in politica estera abbraccia l'idea neo-ottomana dell'odierna Turchia, sempre più pronta a riscoprire il proprio grande passato, facendolo diventare un carattere inconfondibile del proprio futuro.

62 ENHAILI, Aziz, «La Pax ottomana: Une politique en direction du Moyen-Orient et de l'Europe?», in Moyen-Orient, 9/2011, pp. 26-32.

63 DEL ZANNA, Giorgio, I cristiani e il Medio Oriente (1798-1924), cit., p. 198.

64 TASPINAR, Ömer, «Turkey's Middle East Policies: between Neo-Ottomanism and Kemalism», cit., p. 3 .

65 Famoso il discorso tenuto a Sarajevo dal Ministro Davutoğlu il 23/10/2009. Consultabile su URL: < www.setadc.org/multimedia/texts/202-unofficial-transcript-of-foreign-ministerahmed-davutoglus-speech $>$ [Consultato il 15 febbraio 2012]. 


\section{Conclusione}

Il presente lavoro ha voluto analizzare alcuni elementi chiave caratterizzanti l'attuale Turchia ed in particolar modo le sue linee guida in politica estera. L'intento era quello di evidenziare un legame tra l'attuale decennio trascorso sotto la guida del Partito per la Giustizia e Sviluppo (AKP) e il decennio (1983-1992) contraddistinto dall'influente figura di Turgut Özal.

In entrambi i periodi analizzati si ritrova un comune richiamo ad una vecchia idea generatasi all'interno dell'Impero ottomano: l'ottomanismo. Quest'ultimo, dopo un lungo letargo obbligato dalla Repubblica kemalista, riemerge sotto una nuova e moderna elaborazione con il nome di neo-ottomanesimo. Una lettura inedita dell'ottomanismo, questa, che mette in relazione in maniera indissolubile politica identitaria e politica estera, ritenendo lo sviluppo di una comune identità turcoottomana fondamentale per promuovere le ambizioni geopolitiche della Turchia.

Tale politica verte sul recupero non solo dell'eredità ottomana, ma anche del peculiare rapporto con l'universo islamico turco/ottomano caratterizzato, oltre che dall'Islam ufficiale degli ulama, dalla ramificata presenza di confraternite sufi (Nakşibendi, per esempio) e movimenti islamici (Nurcu) considerati i custodi durante gli anni del Kemalismo - del patrimonio culturale ottomano-islamico.

Punto di partenza di quest'analisi sono state le innovative politiche intraprese negli anni Ottanta dal governo Özal, che hanno favorito l'emergere di una nuova idea di società turca caratterizzata da maggiori aperture nel settore economico e in politica estera; sviluppi che accelerarono il processo di rivalutazione e successiva politicizzazione dell'Islam.

Nella seconda parte, invece, si è analizzato l'attuale ruolo della Turchia in campo internazionale dove, seguendo i principi teorizzati dal Ministro degli Esteri Ahmet Davutoğlu nella sua dottrina della "profondità strategica", l'AKP ha saputo costruire l'immagine di una Turchia affidabile e matura, sempre più indispensabile per gli equilibri regionali.

Nella Turchia odierna, tuttavia, permangono diversi limiti, come quello relativo al livello democratico del Paese, che la stessa impostazione neo-ottomana dovrà imprescindibilmente trattare se vorrà coltivare le proprie ambizioni. Il progetto neoottomano, infatti, costringe il governo turco a confrontarsi con situazioni critiche, tra cui le relazioni con Israele, recentemente deterioratesi, e la crescente diffidenza di alcune petromonarchie saudite (Qatar, Arabia Saudita) spaventate dall'ammirazione 
suscitata sulle masse dalla retorica di Erdoğan. Un'altra incognita futura è rappresentata dai rapporti con l'altra potenza regionale forte e in ascesa, quell'Iran che inevitabilmente rappresenterà un ostacolo ai progetti turchi.

A questo si deve aggiungere la storica polarizzazione interna alla società turca tra laici e tradizionalisti islamici che il neo-ottomanesimo non ha fatto altro che accentuare ulteriormente. Sullo sfondo, infine, rimane l'ingombrante ombra dell'esercito e i timori di un suo, al momento poco probabile, nuovo intervento nella vita politica del Paese.

Difficile poter immaginare la Turchia come modello esportabile nei Paesi mediorientali, poiché la specificità turca risiede in molteplici suoi aspetti (culturali, storici, politici, sociali) che la rendono difficilmente imitabile, compreso il carattere del tutto particolare dell'Islam turco.

Detto questo, la presa di coscienza da parte dell'Occidente della forza e del potenziale dell'attuale Turchia, deve aiutare a costruire nuove basi per un rapporto che sia caratterizzato da una minore diffidenza reciproca. Un rapporto nuovo, utile alla Turchia per proseguire la propria maturazione in chiave democratica e di Stato moderno e all'Occidente per cercare un maggiore dialogo con un mondo, come quello musulmano, da cui è ancora considerato troppo ostile. 


\section{* L'autore}

Federico Donelli è laureato in Scienze Politiche e delle Relazioni Internazionali presso l'Università Cattolica del Sacro Cuore di Milano; ha conseguito la Laurea Magistrale in Politica ed Economia del Mediterraneo presso la Facoltà di Scienze Politiche dell'Università degli Studi di Genova. Attualmente è dottorando di ricerca presso l'Università degli Studi di Genova.

URL: < http://www.studistorici.com/progett/autori/\#Donelli >

\section{Per citare questo articolo:}

DONELLI, Federico, «Le radici ottomane della Turchia di Erdoğan », Diacronie. Studi di Storia Contemporanea: Sulle tracce delle idee, 29/12/2012,

URL:<http://www.studistorici.com/2012/12/29/donelli_numero_12/ >

Diacronie Studi di Storia Contemporanea $\beta$ www.diacronie.it

Risorsa digitale indipendente a carattere storiografico. Uscita trimestrale.

redazione.diacronie@hotmail.it

Comitato di redazione: Marco Abram - Jacopo Bassi - Luca Bufarale - Gianluca Canè - Alessandro Cattunar - Alice De Rensis - Barbara Galimberti - Deborah Paci - Fausto Pietrancosta - Matteo Tomasoni - Luca Zuccolo 